\title{
La función pulmonar en sujetos con hemiplejía/hemiparesia crónica. Revisión bibliográfica
}

\author{
Pulmonary function in chronic hemiplegic/hemiparetic patients. A review of the \\ literature
}

\author{
A. Lista Paz y M.L. González Doniz
}

\begin{abstract}
Resumen
Objetivo: Realizar una revisión bibliográfica sobre la función pulmonar y la dinámica costal y diafragmática de los pacientes con hemiplejía/hemiparesia crónica.

Material y métodos: Bases de datos: Medline, Embase y PEDro. Se limitaron las búsquedas por el idioma y se descartaron las cartas al director y las editoriales. No se estableció límite por fecha de publicación. Fueron analizados 10 artículos que satisfacían el objetivo de la revisión.

Resultados: Los sujetos con hemiplejía/hemiparesia crónica muestran un patrón respiratorio restrictivo, con disminución significativa de la máxima ventilación voluntaria y las presiones respiratorias máximas, sumado a una dinámica costal y diafragmática alterada.

Conclusiones: La población analizada muestra limitaciones en los flujos y volúmenes pulmonares y alteraciones en la musculatura respiratoria. Se necesitan futuros trabajos metodológicamente rigurosos, que nos permitan conocer, con un alto grado de evidencia, el alcance del deterioro de la función pulmonar que se da en la hemiplejía/hemiparesia crónica.
\end{abstract}

\begin{abstract}
Objective: To review the literature about pulmonary function and costal and diaphragmatic dynamics in chronic hemiplegic/hemiparetic patients.

Materials and Methods: Databases: Medline, Embase and PEDro. The searches were limited by the language and letters and editorials were ruled out. No deadlines for the publication were established. Finally, 10 articles that adhered to the objective were analyzed.

Results: Patients with a chronic hemiplegia / hemiparesis have a restrictive breathing pattern with a reduction of Maximum Voluntary Ventilation and lower maximum respiratory pressures. Moreover, they have altered costal and diaphragmatic dynamics.

Conclusions: The population analyzed show limited lung flows and volumes and alterations in the respiratory muscles. Future methodologically rigorous research is needed in order to know with a high level of evidence the importance of the deterioration of the pulmonary function within chronic hemiplegia/hemiparesis patients.
\end{abstract}

Palabras clave

Sistema respiratorio; Musculatura respiratoria; Test de función respiratoria; Espirometría; Ictus; Hemiplejía

Keywords

Respiratory System; Respiratory Muscles; Respiratory Function Tests; Spirometry; Stroke; Hemiplegia 


\section{Introducción}

Las enfermedades cerebrovasculares (ECV) constituyen la primera causa de discapacidad y la tercera causa de muerte en los países desarrollados ${ }^{1}$. Dentro de éstas destaca por su alta incidencia el ictus o accidente cerebrovascular $(\mathrm{ACV})^{2}$, que supone la segunda causa de muerte en España, y la primera en algunas comunidades autónomas como Galicia y Extremadura, según los datos publicados por el Instituto Nacional de Estadística (INE) en el $2001^{3}$. En España la incidencia anual del ACV se estima entre los 138 a 200 casos nuevos/año por 100.000 habitantes, lo que equivale aproximadamente a 85.000 casos anuales.

El gran impacto epidemiológico y sociosanitario que supone el ACV ha impulsado el avance en los métodos de rehabilitación de los pacientes que padecen sus consecuencias, abogando por su independencia funcional y su reinserción social.

La evaluación y terapéutica de las complicaciones respiratorias que se producen durante la fase aguda de la enfermedad han sido ampliamente estudiadas, ya que suponen un riesgo vital para el paciente durante las primeras horas de evolución. Así, son conocidos los patrones respiratorios alterados de la primera fase de la enfermedad ${ }^{5,6}$, como taquipnea, respiración de Cheyne-Stokes, riesgo de aspiración ${ }^{7}$, trastornos respiratorios del sueño ${ }^{8,9}$, etc. En el período subagudo también se han realizado algunos estudios de la función pulmonar. Nuzzo et al ${ }^{10}$, en una muestra de 13 pacientes hemipléjicos, encontraron una reducción estadísticamente significativa en la MVV, la Pimax, la CVF y el VEMS. La disminución en las presiones respiratorias máximas también fue hallada por Lanini et al ${ }^{11,12}$ en dos estudios realizados durante el período subagudo de la enfermedad cerebrovascular.

Sin embargo, cuando se trata de pacientes con hemiplejía/ hemiparesia crónica, una de las grandes olvidadas ha sido, y continúa siendo, la función pulmonar, tal vez porque estos pacientes no suelen referir sintomatología respiratoria y las estrategias terapéuticas van dirigidas, sobre todo a disminuir sus discapacidades motoras. La musculatura del tronco recibe inervación bilateral del sistema nervioso central (SNC) a través de los tractos descendentes anteriores y de las interneuronas cruzadas ${ }^{13}$, por lo que la lesión focal de un hemisferio conduce a una afectación contralateral de la musculatura respiratoria ${ }^{12}$. La debilidad y descoordinación de la musculatura abdominal sumada a un suelo pélvico hipotónico imposibilita al paciente para crear presiones positivas intraabdominales suficientes para generar flujos espiratorios eficaces. Ello, sumado a una dinámica alterada del diafragma, y una caja torácica retraída por las contracturas que han quedado en la musculatura del tronco como consecuencia de la espasticidad que caracteriza a estos pacientes, disminuye la capacidad de expansión costal, lo que da lugar a unos volúmenes pulmonares disminuidos ${ }^{14}$.

Según Teixeira-Salmela et al $^{15}$ los pacientes que han sobrevivido a un ACV tienen alrededor de un $40 \%$ menos de capacidad para el ejercicio en comparación con los valores de referencia para su edad y sexo en sujetos sedentarios. Se ha demostrado que el entrenamiento cardiovascular reporta mejoras en este sentido ${ }^{16-18}$, sin embargo, no hay estudios que lo asocien al entrenamiento de la musculatura respiratoria. Por su parte, un reciente ensayo clínico controlado y aleatorizado ha demostrado que el entrenamiento de la musculatura respiratoria en pacientes que han sufrido un ACV tiene resultados positivos sobre los volúmenes pulmonares, la MVV y las presiones respiratorias máximas, así como un aumento sobre la capacidad para el ejercicio, y mejora de la percepción de la disnea y la calidad de vida ${ }^{19}$.

El objetivo del presente trabajo es realizar una revisión bibliográfica sobre el comportamiento de la función pulmonar y la dinámica costal y diafragmática de los pacientes con hemiplejía/hemiparesia crónica, secundaria a una ECV.

\section{Material y métodos}

\section{Estrategia de búsqueda}

Para la elaboración de esta revisión bibliográfica se han empleado las siguientes bases de datos: Medline/PubMed, Embase y PEDro. La elección de las palabras clave para realizar la búsqueda de información se realizó a través del MeSH Database de PubMed. Estas fueron: «Respiratory Function Tests», «Spirometry», «Respiratory System», «Respiratory Physiological Phenomena», «Respiratory Muscles» y «Diaphragm». Cada uno de estos descriptores fueron relacionados, a través del marcador booleano AND, con los siguientes términos: «Stroke», «Hemiplegia» y «Brain Injuries».

En el caso de Medline/Pubmed, también se realizó una búsqueda manual a través de las sugerencias proporcionadas por la propia base de datos, agrupadas bajo el término «citas relacionadas».

Todas las búsquedas fueron limitadas por el idioma (inglés, francés, espa nol, italiano y portugués), descartándose las cartas al director y las editoriales. Sin embargo, no se estableció ningún límite en la 
antigüedad de las publicaciones, por lo que los resultados ofrecidos abarcan hasta el 10 de octubre de 2010 .

\section{Selección de estudios y datos}

Tras leer el abstract de todos los resultados obtenidos, se seleccionaron aquellos artículos que satisfacían el objetivo de este trabajo. Dado que las publicaciones a este respecto son muy escasas y metodológicamente no hay mucha homogeneidad entre ellas, la utilización de criterios de calidad para su selección se vio limitada.

Los estudios que como muestra seleccionaron pacientes en período agudo o subagudo de la ECV fueron descartados de la revisión. Aunque la mayor parte de los autores se refieren a una hemiplejía/hemiparesia crónica cuando han transcurrido al menos 6 meses desde que tuvo lugar el ACV, no existe absoluto acuerdo entre la comunidad científica a este respecto. Por ello, se han seleccionado todos los trabajos en los que sus autores se refieren a la muestra reclutada como pacientes con hemiplejía/hemiparesia crónica.

Finalmente fueron seleccionados diez artículos, todos ellos indexados en Medline/PubMed. Siete de ellos versan sobre la función pulmonar de los pacientes con hemiplejía/ hemiparesia crónica. Salvo un artículo de revisión bibliográfica, todos ellos son estudios observacionales, de entre los cuales dos dispusieron de grupo control. En el estudio de la dinámica costal de este grupo de población se han seleccionado dos trabajos, ambos con grupo control. Por último, se ofrece un artículo sobre la función diafragmática, que también contó con un grupo control.

\section{Resultados}

\section{La función pulmonar en pacientes con hemiplejía/hemiparesia crónica}

La primera referencia que encontramos en la literatura sobre la función respiratoria de los pacientes con hemiplejía crónica data de 1967, de la mano de Haas et al $^{20}$ En una muestra de 19 pacientes con hemiplejía crónica se encontraron los siguientes hallazgos: 1) durante la inspiración máxima se encontró una disminución media de 2,5 pulgadas $(6,35 \mathrm{~cm})$ en la excursión del hemidiafragma afecto en comparación con el lado sano. 2) La CV, tanto en hombres como en mujeres, se vio ligeramente disminuida. 3) Hubo un incremento importante en la CRF en ambos sexos (150\% en hombres y $156 \%$ en mujeres, respecto al valor predictivo). 4) La MVV se encontró ligeramente disminuida en las mujeres, mientras que se obtuvo un llamativo descenso en el grupo masculino (el $73 \%$ respecto al valor de referencia). 5) Los flujos mesoespiratorios $\left(\mathrm{MEF}_{25-75 \%)}\right.$ en ambos sexos se vieron disminuidos cerca de un $50 \%$ respecto del valor de referencia. Estos autores atribuyeron los bajos volúmenes pulmonares a la parálisis de la musculatura inspiratoria del lado afecto, mientras que la debilidad de los músculos espiratorios, sumado al movimiento alterado del diafragma, explicaría el elevado VR. El déficit en los valores de la MVV también quedó justificado por una alteración en la musculatura respiratoria.

En 1978, Odia ${ }^{21}$ sugiere que los pacientes con una hemiplejía de más de tres meses de evolución presentan un patrón ventilatorio restrictivo, que podría tener mucha importancia clínica en la tolerancia al ejercicio físico intenso. Estas conclusiones fueron extraídas del estudio de la función pulmonar llevado a cabo en 20 pacientes con hemiplejía. Se registraron los siguientes valores: la CVF, el VEMS y el PEF. Tras estas mediciones, los pacientes caminaron una distancia de 18,5m tan rápido como pudieron, utilizándose el tiempo en segundos para determinar su estado físico. Los resultados mostraron una reducción significativa tanto de la CVF como del VEMS en ambos sexos, con respecto a los valores predictivos $(p=0,001$ en ambos sexos para la CVF; $p=0,001$ y $p=0,01$ para los valores del VEMS de hombres y mujeres, respectivamente). Sin embargo, no se encontró correlación entre el grado de discapacidad y los valores espirométricos. Dado que la relación VEMS/CVF, así como el PEF fueron normales, los autores apuntaron a la presencia de un patrón ventilatorio de tipo restrictivo.

Fugl-Meyer et al, en $1983^{22}$, arrojaron grandes aportaciones a la evidencia científica de la función pulmonar en pacientes con hemiplejía/hemiparesia crónica, al correlacionar la alteración de la función pulmonar tanto con el grado de afectación motora como con el tiempo transcurrido tras el ACV. Para ello, dispusieron de una muestra de 54 hemipléjicos/hemiparésicos crónicos y un grupo control de 12 sujetos sanos. Los individuos del grupo de ensayo fueron clasificados de acuerdo con su grado de afectación motora, según la escala de Fugl-Meyer ${ }^{23}$ en tres grupos: hemiplejía severa, hemiplejía marcada y hemiplejía moderada/ligera. Los resultados evidenciaron lo siguiente: la CPT, la CV, y la CRF fueron significativamente más bajas $(\mathrm{p}<0,05)$ en los sujetos con hemiplejía severa y marcada, aunque mostraron valores normales en los hemipléjicos moderados/ligeros. El VRE solo mostró una disminución 
estadísticamente significativa en el caso de los sujetos con afectación severa. Tanto la Pimax como la Pemax fueron significativamente menores en todos los grupos de hemipléjicos y la MVV fue menor solo en los sujetos con hemiplejía severa y marcada. En cuanto a la correlación entre el tiempo transcurrido tras el ACV y la disminución de los volúmenes pulmonares y las presiones respiratorias máximas, tan solo la CI demostró dicha relación transcurridos seis meses desde el ACV. Por tanto, entre las conclusiones de este estudio cabe destacar lo siguiente: la disminución de la fuerza espiratoria fue el factor común en todos los pacientes hemipléjicos/ hemiparésicos, acompañada por un detrimento de la CRF, el VRE y la MVV, más pronunciada en los sujetos con mayor afectación neuromuscular. Además, la Pimax se vio disminuida en todos los sujetos, sin embargo, no fue acompañada de una disminución en la CI durante los primeros meses de la enfermedad.

Un año más tarde, Fugl-Meyer y Grimby ${ }^{14}$ realizan una revisión bibliográfica acerca de la función respiratoria en la tetraplejía y la hemiplejía. En lo que al período crónico de la hemiplejía se refiere, hacen mención a los hallazgos encontrados en el estudio de Fugl-Meyer et al de 1983, citado anteriormente, y presumen la siguiente explicación para la existencia de un patrón respiratorio de tipo restrictivo: en algunos pacientes hemipléjicos el descenso del diafragma durante la inspiración, tanto forzada como en reposo, es limitado. Por otra parte, existe un movimiento paradójico de la caja torácica, especialmente durante la tos, ya que el hemitórax afecto se expande, pero tras la maniobra espiratoria no retorna a la posición inicial. Todo ello, sumado a las contracturas que se producen en la musculatura del tronco cuando se ve afectada por la espasticidad que caracteriza a los pacientes hemipléjicos, y la marcada descoordinación y debilidad de la pared abdominal y del suelo pélvico generan tanto un descenso de los volúmenes pulmonares, especialmente los espiratorios, como una disminución de la fuerza espiratoria máxima, ya que el paciente no es capaz de generar la presión intraabdominal positiva suficiente.

En la década de los noventa Annoni et $\mathrm{al}^{24}$ publican un estudio sobre la función respiratoria en pacientes hemipléjicos crónicos, con objetivos similares a los perseguidos por Fugl-Meyer et al, en 1983. ${ }^{22}$ Realizaron una valoración espirométrica a 23 pacientes con una hemiplejía secundaria a un ACV, a una hemorragia intracerebral o bien a una cirugía por tumor cerebral. Existía un rango de tiempo tras el período agudo de la enfermedad desde 2 meses a 10 años. Todos ellos sin historial tabáquico ni patología pulmonar previa. Buscaban relacionar la disfunción respiratoria de estos pacientes con el grado de afectación motora, medido a través de la función del miembro superior pléjico mediante la British Medical Research Council; así como con el tiempo transcurrido tras el período agudo de la enfermedad. Para la consecución del último objetivo los pacientes fueron clasificados en dos grupos, el primero formado por aquellos sujetos con menos de 6 meses de evolución y el segundo por aquellos con más de 6 meses. Entre los resultados de este trabajo destacan los siguientes: 1) la CV solo mostró una disminución estadísticamente significativa en el caso de los sujetos con una función motora del miembro superior afecto de 0-1 medido con la British Medical Research Council, mostrándose independiente del tiempo de evolución de la enfermedad. 2) Tanto la CVF como la CVIF se hallaron claramente disminuidas, aunque sin correlación con el grado de afectación motora, pero sí con el tiempo transcurrido tras el período agudo de la enfermedad, siendo esta disminución estadísticamente significativa solo en el grupo de personas con más de 6 meses de evolución. Las mismas observaciones se encontraron en el VEMS. 3) El PEF y el MEF al 75\% de la CVF fueron significativamente más bajos que en sujetos sanos, guardando relación con el grado de afectación motora del miembro superior pléjico. 4) La relación VEMS/CVF estuvo por encima de la media comparado con sujetos sanos. Este aumento demostró significancia estadística a partir de los 6 meses.

Annoni et al concluyen que existe un patrón ventilatorio restrictivo en los pacientes con hemiplejía crónica, ya que los «valores forzados» (CVF, CVIF, y PEF) se hallan disminuidos, sin embargo, la relación VEMS/CVF se encuentra por encima del valor de referencia, lo que descarta un patrón de tipo obstructivo. Esta disfunción respiratoria explica la disminución de la tolerancia al ejercicio en los pacientes con hemiplejía de larga evolución. Por una parte, la disminución en la CV demuestra la debilidad de la musculatura respiratoria durante los esfuerzos voluntarios. Pero además, los «valores forzados» disminuyen más con el tiempo, por tanto, a la debilidad muscular también se unen alteraciones en la dinámica costal, derivadas del incremento de la espasticidad durante el esfuerzo. Por último, la disminución del PEF indica que estos pacientes tienen una tos menos eficaz que los sujetos sanos.

Doce años más tarde, Narin y Puckree ${ }^{25}$ seleccionaron una muestra de 20 pacientes hemipléjicos con una cronicidad de 2 a 5 años, en los que se midieron una serie de parámetros ventilatorios (VT, FR, V $\mathrm{V}_{\mathrm{E}}$, $\mathrm{VO}_{2}$ y $\mathrm{VCO}_{2}$ ) durante la posición de reposo en sedestación, durante la transferencia de sedestación a bipedestación y durante la bipedestación. Los resultados del estudio mostraron un aumento estadísticamente significativo de todos los parámetros, tanto en la transferencia de sedestación a bipedestación como durante la bipedestación, con respecto a los valores obtenidos en la sedestación. Tras la discusión científica de los datos, estos autores concluyen que existe la necesidad de enfatizar dentro del programa de rehabilitación de los pacientes hemipléjicos la flexibilidad, movilidad, fuerza y resistencia de 
la caja torácica y de los músculos respiratorios, tanto primarios como accesorios, a fin de optimizar la ventilación pulmonar en estos sujetos.

El último estudio existente sobre la función pulmonar en los pacientes con hemiplejía crónica fue publicado en 2005 por Teixeira-Salmela et al ${ }^{15}$ Se comparan las mediciones efectuadas en un grupo de 16 pacientes con hemiplejía de al menos 9 meses de evolución, con las obtenidas en 19 sujetos sanos que actúan como grupo control. Entre los hallazgos más importantes se encuentran los siguientes: disminución estadísticamente significativa de la Pimax y la Pemax, así como una mayor contribución de la caja torácica en el ciclo respiratorio al borde de la significación estadística $(p=0,05)$ que podría sugerir una baja participación de la musculatura abdominal. Debido a que los músculos abdominales no actúan solo como agonistas durante la espiración activa sino que también juegan un papel importante durante la inspiración, contribuyendo directamente a la acción del diafragma con el mantenimiento del tono de la pared abdominal, tanto la fuerza como el tono de los músculos abdominales pueden evitar un excesivo acortamiento del diafragma después de cada contracción, permitiéndole al músculo inspiratorio por excelencia trabajar en una posición más favorable de su curva longitud-tensión. Así pues, la debilidad de la musculatura abdominal puede afectar a la sinergia entre los músculos abdominales y el diafragma, impidiendo la capacidad del diafragma para generar la fuerza suficiente para una ventilación óptima ${ }^{26}$.

Teixeira-Salmela et al señalaron que el reducido tamaño de la muestra no permitió encontrar diferencias estadísticamente significativas entre los patrones ventilatorios del grupo experimental y el grupo control.

En la tabla 1 se ofrece una visión global de los estudios observacionales que evalúan la función pulmonar de los pacientes con hemiplejía/hemiparesia crónica.

Tabla 1 La función pulmonar en los pacientes con hemiplejía/hemiparesia crónica. Estudios observacionales

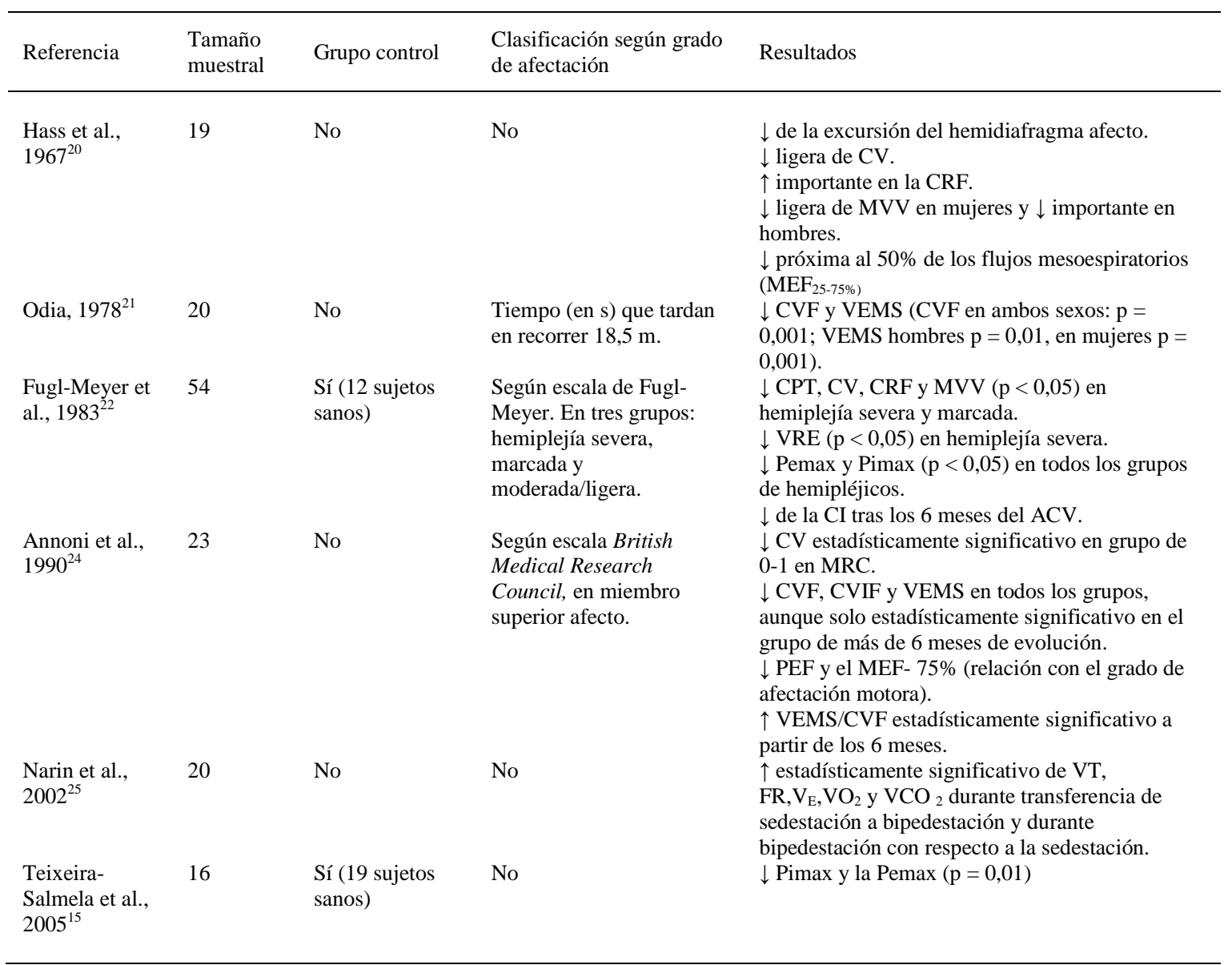




\section{La dinámica costal y diafragmática en los pacientes con hemiplejía/hemiparesia crónica}

Como hemos visto anteriormente, algunas investigaciones han evidenciado la presencia de un patrón respiratorio restrictivo en los pacientes con hemiplejía/hemiparesia crónica. Una explicación plausible para tal hallazgo pasa por corroborar la alteración en la dinámica costal de este grupo de población.

En 1966, Fluck ${ }^{27}$ llevó a cabo un estudio con una muestra de 57 sujetos con hemiplejía de una evolución media de dos años y medio, y un grupo control de 22 sujetos sanos. Se objetivaron los movimientos costales (a nivel superior e inferior de la caja torácica) durante la respiración en reposo, la respiración profunda voluntaria y la respiración profunda involuntaria. Durante la respiración en reposo se observó una reducción media del 9,2\% $(\mathrm{p}<0,05)$ en la región costal superior del hemitórax del lado plégico. La reducción del movimiento en el lado afecto fue estadísticamente significativa tanto en la región costal superior $(16,9 \%, \mathrm{p}<0,01)$ como en la región costal inferior $(9,5 \%, \mathrm{p}<0,02)$, durante la respiración voluntaria. Sin embargo, durante la respiración involuntaria los movimientos costales se registraron simétricos en ambos grupos, excepto en la región costal inferior derecha del grupo control, que mostró un movimiento significativamente más amplio $(\mathrm{p}<0,01)$ que el lado izquierdo.

Fluck et al, basándose en los hallazgos de su estudio sugieren que la inervación supramedular de los músculos de la caja torácica es asimétrica durante la respiración en reposo y la respiración voluntaria, pero no durante la respiración involuntaria. Además, estos resultados podrían apoyar la idea de otros autores de que el elevado tono muscular del lado plégico, así como las retracciones presentes en la caja torácica impiden un movimiento completo de este hemitórax ${ }^{28}$.

En el año 2001, Ogiwara y Ogura ${ }^{29}$ estudiaron el movimiento torácico antero-posterior durante la respiración profunda voluntaria en 30 sujetos con una hemiplejía residual, en comparación con un grupo control constituido por el mismo número de individuos. Se registró una diferencia estadísticamente significativa $(\mathrm{p}<0,01)$ entre la excursión antero-posterior del hemitórax afecto y el sano de los pacientes hemipléjicos. Estos autores sugieren una restricción en los movimientos de la caja torácica tras el ACV.

En este estudio solo se midieron los movimientos costales en el plano anteroposterior. Se necesitan futuras investigaciones en esta población diana que analicen la dinámica costal como movimiento tridimensional.

En la tabla 2 se ofrece un cuadro conceptual de los estudios analizados sobre la dinámica costal en los pacientes con hemiplejía/hemiparesia crónica.

Tabla 2 Comportamiento de la dinámica costal en pacientes con hemiplejía/hemiparesia crónica

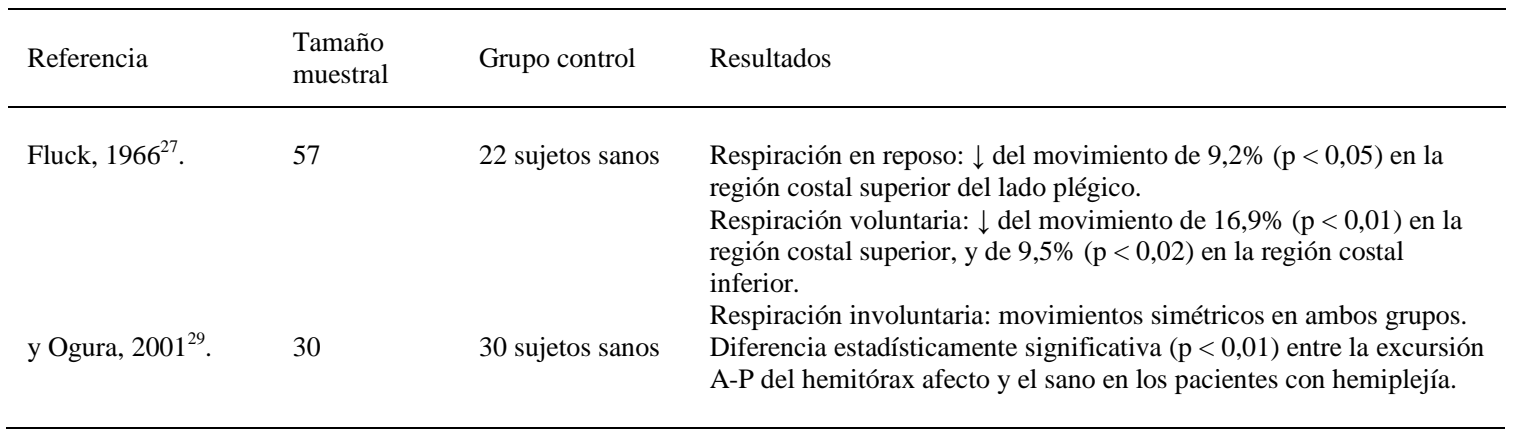

Para dar una visión amplia del estado de la salud respiratoria de los pacientes que padecen hemiplejía/hemiparesia crónica es también obligado hacer referencia a los trabajos publicados sobre la musculatura respiratoria.

Durante la fase aguda del ACV, algunos estudios llevados a cabo a través de electromiografía y ultrasonografía han demostrado una clara disminución en la actividad de la musculatura del hemitórax parético durante la ventilación voluntaria ${ }^{30,31}$. Un estudio más reciente ha demostrado, a través de estimulación magnética transcraneana, una clara asociación entre la debilidad de la musculatura respiratoria y el accidente cerebrovascular de tipo isquémico ${ }^{32}$.

Respecto a las alteraciones en la posición y la función del diafragma durante la respiración en los sujetos que han sufrido un ACV, ya en el año 1969, Korczyn et al $^{33}$ publicaron un trabajo en el que se compararon las radiografías de 190 pacientes hemipléjicos/hemiparésicos, tomadas en posición supina durante la inspiración profunda, con las de 100 sujetos sanos, a fin de evidenciar la posición alterada del 
diafragma del lado afecto. Los resultados demostraron una mayor elevación del hemidiafragma afecto en comparación con el no afecto ( $\mathrm{p}<0,01)$. Del mismo modo, al comparar al grupo de estudio con el grupo control se halló una diferencia significativa en la elevación diafragmática $(\mathrm{p}<0,02)$, siendo mayor en los primeros.

\title{
Discusión y conclusiones
}

Los estudios realizados sobre el comportamiento de la función pulmonar en sujetos con hemiplejía/hemiparesia crónica apuntan a un patrón respiratorio restrictivo, sumado a una dinámica costal y diafragmática alterada.

Cabe destacar que todas las investigaciones realizadas encontraron una disminución significativa de la MVV y las presiones respiratorias máximas, lo cual indica que existe un déficit en la fuerza y la resistencia de la musculatura respiratoria. Sin embargo, encontramos otros datos en los que existen discrepancias. Haas et $\mathrm{al}^{20}$ obtuvieron un importante aumento de la CRF, mientras que Fugl-Meyer et $\mathrm{al}^{22}$, encontraron justamente lo contrario. Odia ${ }^{21}$ no obtuvo una correlación entre el grado de discapacidad motora y los valores espirométricos, lo que contrasta con los resultados de los estudios de Fulgl-Meyer et $\mathrm{al}^{22}$, y Annoni et $\mathrm{al}^{24}$, quienes demostraron una correlación significativa entre la alteración de la función pulmonar y el grado de afectación motora, así como con el tiempo transcurrido tras el ACV. Sin embargo, estos estudios no son comparables entre sí, pues utilizaron medidas de valoración muy diferentes para establecer el grado de afectación de los sujetos reclutados como muestra.

Dichas contradicciones se explican, en gran medida, por la heterogeneidad metodológica de estas investigaciones, en cuyas publicaciones existen importantes vacíos de información, que deben ser tenidos en cuenta. En la mayoría de los estudios ${ }^{15,20,21,25}$ desconocemos si los sujetos reclutados eran o no fumadores o ex fumadores; en otros $\operatorname{casos}^{20,25}$ no sabemos qué grado de afectación motora tenían los pacientes muestreados y en un estudio ${ }^{20}$ no tenemos constancia del tiempo transcurrido tras el ACV. Además, el diseño de algunos estudios ${ }^{20,21,24}$ no incluyó un grupo control con el que comparar los resultados de las mediciones, sino que se expresaron como porcentajes con respecto a los valores de referencia obtenidos mediante ecuaciones predictivas. Sin embargo, en el caso de Annoni et $\mathrm{al}^{24}$, no se especifica qué ecuación predictiva se utilizó. Otro problema metodológico lo encontramos en la investigación llevada a cabo por Haas et $\mathrm{al}^{20}$, quienes no aportaron el valor de «p», por lo que los resultados no son extrapolables a la población diana de estudio, ya que desconocemos su significancia estadística. Por último, en el caso de Teixeira-Salmela et $\mathrm{al}^{15}$, todas las mediciones fueron tomadas en posición supina por lo que los resultados no son extrapolables a la posición de sedestación, en la que normalmente se efectúan las pruebas espirométricas.

Según los artículos analizados, los pacientes con hemiplejía/ hemiparesia crónica presentan una limitación en los flujos y volúmenes pulmonares, así como una alteración de la dinámica costal y diafragmática y disminución de la fuerza y resistencia de la musculatura respiratoria. Es necesario el planteamiento de un estudio, metodológicamente riguroso, que nos permita corroborar, con un alto grado de evidencia científica, los hallazgos mencionados, a fin de poder en una línea de investigación posterior evaluar la efectividad de un programa de fisioterapia respiratoria como parte de la rehabilitación de este grupo de población.

\section{Conflicto de intereses}

Los autores declaran no tener ningún conflicto de intereses.

\author{
Anexo 1. Abreviaturas \\ CI (IC) $=$ Capacidad Inspiratoria. \\ CRF $($ FRC $)=$ Capacidad Residual Funcional \\ $\mathrm{CV}(\mathrm{VC})=$ Capacidad Vital \\ CVF $($ FVC $)=$ Capacidad Vital Forzada \\ CVIF $($ FIVC) $=$ Capacidad Vital Inspiratoria Forzada. \\ $\mathrm{FR}=$ Frecuencia Respiratoria . \\ MEF25-75\%=Flujo Espiratorio Forzado entre el 25 y el $75 \%$ de la CVF. \\ MVV= Máxima Ventilación Voluntaria \\ $\mathrm{PEF}=$ flujo espiratorio máximo. \\ Pemax $(\mathrm{PEM})=$ Presión Espiratoria Máxima. \\ Pimax $(\mathrm{PIM})=$ Presión Inspiratoria Máxima. \\ $\mathrm{VCO}_{2}=$ producción de dióxido de carbono
}


$\mathrm{VE}=$ ventilación minuto.

VEMS (FEV1)=Volumen máximo espirado en el primer segundo.

$\mathrm{VO}_{2}=$ consumo de oxígeno.

$\mathrm{VR}=$ Volumen Residual.

VRE $($ ERV $)=$ Volumen de Reserva Espiratoria.

$\mathrm{VT}=$ Volumen Tidal.

\section{Bibliografía}

1. Calderón CV, Martí-Fàbregas J, Martí-Vilalta JL. Patología vascular cerebral. En: Serra Gabriel MR, Díaz Petit J, De Sande Carril ML, editores. Fisioterapia en neurología, sistema respiratorio y aparato cardiovascular. Barcelona: Masson; 2005. p. 43-51.

2. Baer G, Durward B. Ictus. En: Stokes M, editor. Fisioterapia en la rehabilitación neurológica. Madrid: Mosby; 2006. p. 81-111.

3. Durán MA. Informe sobre el impacto social de los enfermos dependientes por ictus. INFORME ISEDIC Madrid. 2004.

4. Pérez Sempere A. Cerebrovascular morbidity in Spain: incidence and prevalence. Rev Neurol. 1999;29:879-81.

5. Lee MC, Klassen AC, Resch JA. Respiratory pattern disturbances in ischemic cerebral vascular disease. Stroke. 1974;5:612-6.

6. Rowat A M, Wardlaw JM, Dennis MS. Abnormal breathing patterns in stroke: relationship with location of acute stroke lesion and prior cerebrovascular disease. Neurol Neurosurg Psychiatry. 2007;78:277-9.

7. Smith Hammond CA, Goldstein LB, Horner RD, Ying J, Gray L, González-Rothi L, et al. Predicting aspiration in patients with ischemic stroke: comparison of clinical signs and aerodynamic measures of voluntary cough. Chest. 2009;135:769-77.

8. Rola R, Wierzbicka A, Wichniak A, Jernajczyk W, Richter P, Ryglewicz D. Sleep related breathing disorders in patients with ischemic stroke and transient ischemic attacks: respiratory and clinical correlations. J Physiol Pharmacol Suppl. 2007;58:575-82.

9. Hermann DM, Bassetti CL. Sleep-related breathing and sleep-wake disturbances in ischemic stroke. Neurolog. 2009;73:1313-22.

10. Nuzzo NA, Bronson LA, Massery M. Respiratory muscle strength and endurance following a CVA. J Neurol Phys Ther. 1999;23:25-7.

11. Lanini B, Gigliotti F, Coli C, Bianchi R, Pizzi A, Romagnoli I, et al. Dissociation between respiratory effort and dyspnea in a subset of patients with stroke. Clin Sci. 2002;103:467-73.

12. Lanini B, Bianchi R, Romagnoli I, Coli C, Binazzi B, Gigliotti F, et al. Chest wall kinematics in patients with hemiplegia. Am J Respir Crit Care Med. 2003;168:109-13.

13. Kuypers HG. Anatomy of descending pathways. En: Broks VB, editor. Handbook of physiology, section 1: The Nervous System, Motor Control, II. Bethesda MD: American Physiological Society; 1981. p. 597-666.

14. Fugl-Meyer AR, Grimby G. Respiratory in tetraplegia and in hemiplegia: a review. Int Rehabil Med. 1984;6:18690.

15. Teixeira-Salmela LF, Parreira VF, Britto RR, Brant TC, Inácio ÉP, Alcántara TO, et al. Respiratory pressures and thoracoabdominal motion in community-dwelling chronic stroke survivors. Arch Phys Med Rehabil. 2005;86:1974-8.

16. Macko RF, Ivey FM, Forrester LW, Hanley D, Sorkin JD, Katzel LI, et al. Treadmill exercise rehabilitation improves ambulatory function and cardiovascular fitness in patients with chronic stroke: a randomized, controlled trial. Stroke. 2005;36:2206-11.

17. Pang YC, Eng JJ, Dawson AS, Gylfadóttir S. The use of aerobic exercise training in improving aerobic capacity in individuals with stroke: a meta-analysis. Clin Rehabil. 2006;20:97-111.

18. Lennon O, Carey A, Gaffney N, Stephenson J. A pilot randomized controlled trial to evaluate the benefit of the cardiac rehabilitation paradigm for the non-acute ischaemic stroke population. Clin Rehabil. 2008;22:125-33.

19. Sutbeyaz ST, Koseoglu F, Inan L, Coskun O. Respiratory muscle training improves cardiopulmonary function and exercise tolerance in subjects with subacute stroke: a randomized controlled trial. Clin Rehabil. 2010;24:24050 .

20. Haas A, Rusk HA, Pelosof H, Adam JR. Respiratory function in hemiplegic patients. Arch Phys Med Rehabil. 1967;48:174-9.

21. Odia GI. Spirometry in convalescent hemiplegic patients. Arch Phys Med Rehabil. 1978;59:319-21.

22. Fugl-Meyer AR, Linderholm H,Wilson AF. Restrictive ventilator dysfunction in stroke: its relation to locomotor function. Scand J Rehabil Med Suppl. 1983;9:118-24.

23. Fugl-Meyer AR. Post-stroke hemiplegia, Assessment of physical properties. Scand J Rehabil Med Suppl. $1980 ; 84$.

24. Annoni JM, Ackermann D, Kesselring J. Respiratory function in chronic hemiplegia. Int Disabil Studies. 1990;12:78-80.

25. Narin S, Puckree T. Pulmonary function in hemiplegia. Int J Rehabil Res. 2002;25:57-9.

26. De Troyer A, Estenne M. Functional anatomy of respiratory muscles. Clin Chest Med. 1998;9:175-92.

27. Fluck DC. Chest movements in hemiplegia. Clin Sci. 1966;31:383-8.

28. Kolb LC, Kleyntjens F. A clinical study of the respiratory movements in hemiplegia. Brain. 1937;60:259-74. 
29. Ogiwara S, Ogura K. Antero-Posterior Excursion of the Hemithorax in Hemiplegia. J Phys Ther Sci. 2001;13:115.

30. De Troyer A, Zegerdes De Beyl D, Thirion M. Function of respiratory muscles in acute hemiplegia. Am Rev Respir Dis. 1981;123:631-2.

31. Cohen E, Mier A, Heywood P, Murphy K, Boultbee J, Guz A. Diaphragmatic movement in hemiplegic patients measured by ultrasonography. Thorax. 1994;49:890-5.

32. Harraf F, Ward K, Man W, Rafferty G, Mills K, Polkey M, et al. Transcranial magnetic stimulation study of expiratory muscle weakness in acute ischemic stroke. Neurology. 2008;71:2000-7.

33. Korczyn DA, Hermann G, Don R. Diaphragmatic involvement in hemiplegia and hemiparesis. J Neurol Neurosurg Psychiat. 1969;32:588-90. 